\title{
Clinical Review of Current Techniques of Magnetic Resonance Imaging of Atherosclerosis
}

\author{
Joy Cambe ${ }^{1}$, Sylvia Biso ${ }^{1,3 *}$, Nadia Alie ${ }^{1}$ and Venkatesh Mani ${ }^{1,2}$ \\ ${ }^{1}$ Translational and Molecular Imaging Institute, Icahn School of Medicine at Mt. Sinai, New York, New York, 10029, USA \\ ${ }^{2}$ Department of Radiology, Icahn School of Medicine at Mt. Sinai, New York, New York, 10029, USA \\ ${ }^{3}$ Department of Internal Medicine, Albert Einstein Medical Center, Philadelphia, Pennsylvania, USA
}

\begin{abstract}
Cardiovascular disease (CVD), notably atherosclerosis, is the leading cause of morbidity and mortality worldwide commonly caused by thrombotic occlusion of a vulnerable plaque. Early assessment of atherosclerotic lesions is an important diagnostic goal in order to decrease the coronary artery siases burden. The purpose of this article is to review current MRI techniques on atherosclerosis and explore their clinical applications. First, this article will review the pathogenesis of atherosclerosis and describe various vulnerable plaque features i.e. intraplaque hemorrhage, lipid rich necrotic core, thin fibrous caps, neovascularization, and plaque inflammation. A comparison of different noninvasive in vivo imaging of atherosclerosis, specifically ultrasound, computer tomography, and magnetic resonance imaging will be discussed. This article will argue that MRI is best suited for detecting early plaque lesions. Next, the current MR imaging techniques in atherosclerosis will be introduced. Then this article will examine the clinical impact of MRI on atherosclerotic burden based on their vascular location. Lastly, new strategies in MRI imaging of atherosclerosis will be revealed.
\end{abstract}

Keywords: MRI; Atherosclerotis; Magnetic resonance imaging; Plaques; Clinical applications

\section{Introduction}

Cardiovascular disease (CVD) is the leading cause of morbidity and mortality worldwide, commonly caused by thrombotic occlusion of a vulnerable plaque. An estimated 83.6 million American adults ( $>1$ in 3) have 1 or more types of CVD. Of these, 42.2 million are estimated to be $\geq 60$ years of age. By $2030,40.8 \%$ of the US population is projected to have some form of CVD [1]. CVD, notably atherosclerosis, is a major contributing factor to the total disease burden worldwide. Atherosclerosis is systemic vascular disease that primarily affects the medium and large vessels and usually manifests by ischemic complications. The diverse clinical presentation of atherosclerosis depends on the vascular beds affected. The severity of the clinical manifestations varies widely. Some patients do not experience any clinical manifestations for years but exhibit evidence of chronic atherosclerotic disease only discovered post mortem [2]. Many others experience sudden clinical events such as ischemic stroke, myocardial infarction, and sudden cardiac death [3-5]. These atherosclerotic lesions that are prone to clinical events have been termed "vulnerable" or "high risk" plaques. These vulnerable plaques are cause by rupture of atherosclerotic plaques ensuing thromboembolic events.

\section{Pathogenesis of Atherosclerosis}

Atherosclerosis is characterized by the thickening of the arterial wall resulting in the formations of an atherosclerotic plaque. The etiology of atherosclerosis is influenced by the complex interactions of an individual's biochemical predisposition and acquired risks factors. The arterial wall thickens to form an atherosclerotic plaque, a chronic process involving cholesterol deposition, inflammation, extracellularmatrix formation and possible thrombosis [6]. The disease remains asymptomatic until the plaques either cause obstruction of blood flow or the plaque matrix give way to plaque rupture triggering vessel occlusions and clinical signs of ischemia. Upon injury to the arterial intima oxidized low-density lipoproteins (LDL)-considered the precursor of atherosclerosis- induce inflammatory response which is believed to play an important role in plaque progression and formation of plaque lipid rich necrotic core and fibrous cap $[7,8]$.

The American Heart Association (AHA) classification grades atherosclerotic lesions based on the lesions type's composition. Atherosclerosis begins when said oxidized LDL activate expression of adhesion molecules, such as vascular cell adhesion moledule-1 (VCAM1) and E-selectins, from the endothelial cells. Monocytes migrate from the bloodstream into the wall of the artery and become macrophages. These macrophages deposit fatty materials and accumulate in the inner lining of the arteries over time. Atherosclerotic lesion progress from initial infiltrates of these macrophages and the appearance of foam cells (Type I) to the lipid-laden smooth muscle cells lesions (Type II) that show gross visualization of fatty streaks characterized by layers of macrophage foam cells and lipid deposition within intimal smooth muscles and heterogeneous droplets of extracellular lipids. Type III lesions are intermediate lesion demonstrating morphological and chemical transitions to advanced lesions [9].

Type IV lesions are the first of the advanced lesions characterized by extracellular lipid accumulation known as the lipid core. The lesion progresses to develop a fibrous cap on top of the lipid core (Type Va), followed by vessel wall calcifications (Type $\mathrm{Vb}$ ), then fibrosis (Type $\mathrm{Vc}$ ), and finally Type VI lesions with fissures, hematoma, or thrombosis [10]. Progressively an atherosclerotic lesion can develop new vascular

*Corresponding author: Sylvia Biso, Translational and Molecular Imaging Institute, Icahn School of Medicine at Mt. Sinai, New York, New York, 10029, USA, Tel: 011-1212-824-8454; Fax: 011-1-646 -537-9689; E-mail: BisoSylv@einstein.edu

Received September 30, 2015; Accepted November 07, 2015; Published November 15, 2015

Citation: Cambe J, Biso S, Alie N, Mani V (2015) Clinical Review of Current Techniques of Magnetic Resonance Imaging of Atherosclerosis. J Vasc Med Surg 3: 227. doi:10.4172/2329-6925.1000227

Copyright: ( $) 2015$ Cambe J, et al. This is an open-access article distributed unde the terms of the Creative Commons Attribution License, which permits unrestricted use, distribution, and reproduction in any medium, provided the original author and source are credited. 
connections in the vasa vasorum. Hypoxic stresses provoke apoptotic process and proliferations of new blood vessels into the atherosclerotic wall [11]. This process, known as neovascularization, is governed by endothelial cells response to hypoxic conditions in the tunica intima [12]. It has been documented that plaque neovascularization is increased in ruptured atherosclerotic lesions. Studies have indicated that vulnerability of those plaques may be related to plaque composition rather than the degree of luminal narrowing and that cellular and extracellular composition of atherosclerotic plaques are the major determinants of plaque stability [13].

\section{Characterization of the Atherosclerotic Plaque}

Current clinical management of atherosclerosis relies on two things: degree of luminal narrowing and patient symptomatology. These two determining factors of the progression of atherosclerosis can be most evidently depicted in the case of carotid artery plaques and corresponding cerebrovascular events. Symptomatic patients with high-grade carotid stenosis are at risk for cerebral infarction and are aggressively treated with carotid endarterectomy [14]. Degree of arterial occlusion, however, is not enough to detect patients at risk for cerebrovascular events. It has been shown that patients with less than $70 \%$ carotid stenosis experience and are at risk of stroke [5]. This finding can be attributable to occult atherosclerotic plaque burden and acute arterial occlusion from plaque rupture. Common diagnostic methods, such as angiography, that depend on luminal narrowing cannot accurately measure plaque burden. Arterial walls remodel as a compensatory mechanism to accommodate progressing atherosclerotic plaque and this allows atheromas to increase in size and be displaced outwards before causing any significant stenosis [4]. Arterial occlusion leading to ischemic events can also be caused by plaque rupture with superimposed thrombosis. Plaque morphology affects the risk of plaque disruption, even more than plaque size [3]. Accurate and reliable measurement and characterization of atherosclerotic plaques are, therefore, essential in assessing patients at risk for clinical events.

Determining plaque morphology provides a way of assessing and predicting plaque rupture. Plaque characteristics that contribute to its instability are rupture of the fibrous cap, larger lipid-rich necrotic core, presence of intra-plaque hemorrhage, inflammation of the cap, neovascularization, and irregular superficial calcification of the plaque [3,15-17]. To improve the clinical application of these findings, GuryPaquet et al. studied the main components contributing to plaque instability and created a new imaging score for symptomatic plaque assessment. The HULC (hemorrhage, ulceration and lipid core) scoring strikes a balance between sensitivity and specificity of the various factors and is a promising method for a standardized symptomatic plaque assessment [18]. Adventitial vasa vasorum microvessels contribute to inflammation, intra-plaque hemorrhage, and lipid deposition which lead to plaque instability, rupture and subsequent ischemic events [19]. Among the different plaque components, intra-plaque hemorrhage emerged as the most specific factor in predicting plaque instability. Ulceration or rupture of the fibrous cap and lipid-rich necrotic core, on the other hand, showed higher sensitivity [18].

In clinical practice, early identification and characterization of vulnerable atherosclerotic lesions that are likely to lead to clinical events remains a challenge. Consequently, there is a clinical need for the detection of these vulnerable plaques prior to the development of complications. Although the reference standard for radiological detection of severe atherosclerotic vessel wall changes is still confined to arterial luminal diameter, there is growing evidence that offer new imaging techniques that provide information on plaque composition and biological processes associated with plaque progression and destabilization. In due course, these new imaging techniques which aim to detect atherosclerotic lesions in the asymptomatic phase of the disease may potentially reduce atherosclerotic burden- in tandem decrease the morbidity and mortality of cardiovascular disease.

\section{Non-invasive In vivo Imaging Modalities}

Analysis of the composition of atherosclerotic plaque and accurate measurement of plaque burden will improve patients' embolic risk stratification, prevention, and monitoring of treatment. Patients need a screening tool to help detect early lesions, characterize and locate plaques, monitor atherosclerosis progression or regression, and assess their risk for future cardiovascular outcomes. This is especially true for asymptomatic patients with low-grade stenosis where current clinical standards do not address the possibility of plaque disruption, but rely on the degree of stenosis alone in management decisions. An ideal screening procedure must be accurate, reliable, and have a high sensitivity index in detecting vulnerable atherosclerotic plaques. Furthermore, it must have an excellent cost-benefit ratio, guarantees patient safety, and is readily available. Non-invasive in vivo imaging modalities meet these criteria [20]. Non-invasive procedures have the advantage over the more commonly used angiography procedure in that they do not involve percutaneous access to the vascular system. Currently, the most commonly studied non-invasive in vivo imaging modalities to assess atherosclerotic plaque are ultrasound, computed tomography scan, and magnetic resonance imaging.

Ultrasound uses sound waves to visualize atherosclerotic plaques and to evaluate blood flow. The strengths of transcutaneous ultrasound are it is relatively inexpensive and widely available. It has the capability to demonstrate flow obstruction secondary to stenosis in carotid arteries. B-mode ultrasound imaging is commonly used to directly visualize atherosclerotic plaques, but due to the signal attenuation vessel wall imaging has been limited to shallow vascular beds. B-mode ultrasound can provide a measure of carotid intima-media thickness (IMT) which is a common biomarker used for cardiovascular health. Increase in carotid intima-media thickness is a strong predictor for future vascular events [21]. However, its disadvantages are it is userdependent, limited by heavily calcified plaque, and restricted to the assessment of superficial vessels.

Although this section discusses non-invasive imaging, it is also important to mention that intra-vascular ultrasound (IVUS) is an invasive sonographic modality that has been used in the assessment of coronary plaque. IVUS permits visualization of coronary arteries via B-mode ultrasound using a small transducer attached to a catheter assess the plaque burden. IVUS demonstrated high predictive accuracies of $87.1 \%$ for fibrous, $87.1 \%$ for fibro-fatty, $88.3 \%$ lipid-rich necrotic core, and $96.5 \%$ for dense calcium when compared to plaque specimens from atherectomy. A major challenge with ultrasound techniques is low reproducibility and high measurement variability [6]. Furthermore, IMT does not provide direct information about focal disease or plaque formation and there is a decreased classification accuracy of ultrasound in the presence of calcifications and thrombus. Other modalities, such as MRI demonstrate extensive validations, higher reproducibility, and the ability to monitor the subjects over a period of time which is better suited for plaque quantification and serial analysis.

Another imaging modality, computer tomography (CT), uses ionizing radiation to produce images. Plaque imaging via electron beam CT and multi-detector CT has been used to stratify the risk of future cardiac events by use of the coronary calcium score (CAC). CAC 
helps to predict obstructive coronary disease. In a study of 1851 patients who undergone CT angiography and CAC, it showed to have a high negative power (98\%) between a negative CAC score (no calcifications) and no obstruction on angiography [6]. Another appealing factor is readily available and less expensive than magnetic resonance imaging [22].

It is a less frequently used imaging technique in detecting atherosclerotic plaque because of its many disadvantages. CT generates poor soft tissue images, produces artifacts in calcified plaques, has subpar delineation of plaque components, and exposes patients to ionizing radiation that increases their risk for cancer. This modality is not effective for detecting other components of vulnerable plaques: thin-cap fibroatheromas and presence of inflammatory cells [23]. Noncalcified coronary plaques have been detected and classified by CTA but supported by histological validation is lacking [24-27]. Compared to ultrasound and MRI, radiation exposure from CT is a concern for screening and serial imaging. Motion artifacts are also a limitation for CT imaging, particularly in studies on coronary vessels. Conditions like arrhythmias and high cardiac rates are a challenge even with faster 64 slice CT scanners. Blooming artifacts from extensive calcification can lead to over-estimation of lesion severity [28].

MRI has emerged as a novel modality for detecting and characterizing atherosclerotic plaques. Research and clinical applications of MRI characterization of atherosclerosis has established the diagnostic value of MRI in assessment of plaque burden. A patient is placed on a strong magnetic field and an MRI image is produced from the emitted radiofrequency signals of protons. It has outstanding soft tissue contrast and delineation of plaque components. Plaque characterization is reproducible and has been validated in numerous studies. MRI imaging has also been employed in assessing large vessels such as the aorta [29] as well as smaller ones such as peripheral arteries [30]. It does not expose patients to radiation, has satisfactory spatial resolution, and can be used for serial imaging. Since it takes a longer time to produce an image, respiratory and cardiac movements interfere with the quality of images. Thus, it has limited application in coronary arteries [31]. There is also need for a standardized protocol to be established for the analysis of plaque characteristics. In addition, not all patients are eligible for an MRI scan, particularly those who have pacemakers and those who are claustrophobic. Table 1 compare $s$ the imaging capacities of ultrasound, CT, and MRI and their respective imaging capacities. MRI appears to be the leading non-invasive in vivo imaging modalities to characterize atherosclerotic plaques.

\section{Different MRI Imaging Modalities of Atherosclerosis}

MRI imaging of atherosclerotic plaques is based on the signal intensity and morphology of the plaque on multiple contrast weightings. The signal intensity of these plaques varies according to the proton density (PD) and relaxation time (T1 and T2). The multicontrast images, both bright blood (i.e. time-of-flight) and black blood (i.e. T1W, T2W, PDW with blood-flow suppression) can be used complimentary for visualization of various plaque components such as fibrous tissue, hemorrhage, and dense calcifications. Bright-blood imaging techniques suppress the signal from the surrounding tissues and enhance luminal signals. Therefore, it is primarily applied for evaluation of luminal stenosis. Whereas black-blood imaging attenuates luminal signals for better visualization of vessel wall and provides better outline of the luminal surface and identification characteristics such as intraplaque hemorrhage or fibrous cap rupture. MRI offers versatility to examine different components of atherosclerotic plaques through diffusion, contrast uptake, dynamic contrast permeability, and magnetization transfer. This section aims to provide an overview of the various MRI modalities currently used to in the study of atherosclerotic plaques.

\section{Magnetic Resonance Angiography with Time-of-Flight Sequence}

Magnetic resonance angiography (MRA) with time-of-flight sequences (TOF) provides the capability to measure luminal narrowing. The lumen appears bright and blood flow obstructions can be readily apparent. TOF can aid in assessment of fibrous cap thickness and integrity because it can delineate the lumen shape and internal borders of the plaque. However, this technique cannot separate plaque components or measure the amount of plaque. Black-blood inversion technique has been developed to address this and aid in arterial wall thickness and plaque analysis. Gupta et al. demonstrated that assessment of embolic risk could be made more available to most centers using 3D TOF MRA and standard neck coils. 3D TOF MRA was used to assess intra-plaque hemorrhage (IPH) in this study. Figure 1 shows MRI of intraplaque hemorrhage in T1W, PDW, and T2W imaging and portrays the various vulnerable plaque components. Intraplaque highintensity signal (IHIS), defined as higher than $50 \%$ signal intensity than skeletal muscle, was employed as a surrogate marker for IPH. Patients were scanned using standard quadrature neck array coils, instead of specialized surface carotid coils. Their findings showed that patients with 70 to $99 \%$ carotid stenosis and IHIS are $14 \%$ more likely to have had prior ischemic event than those who have high-grade stenosis and no IHIS. Having shown that patients with IHIS and carotid stenosis are associated with cerebrovascular events, the more widely accessible 3D TOF MRA using standard neck coils has the potential to be used to evaluate for future cerebrovascular risk [32].

For other plaque characteristics that indicate instability, it appears that TOF offers poor visualization of these plaque components. In a study by Etesami et al., TOF MRA missed a third of plaque ulcers that were detected using contrast-enhanced magnetic resonance angiography (CE-MRA). TOF ulcer detection are easily affected by ulcer position, distance to stenosis, and neck-to-depth ratio [33]. Plaque ulcers in TOF images can also be mistaken as IPH. Blood turbulence within the plaque craters has signal intensity similar to IPH and may result to false-positives [34].

Another disadvantage of unenhanced TOF is overestimation of carotid stenosis in areas of turbulent blood flow [35]. TOF is also limited by motion and flow-associated artifacts. Patient movement results to blurring between tissue interfaces. An example of flowrelated artifact is decreased clarity in areas of luminal narrowing [36].

\begin{tabular}{|c|c|c|c|}
\hline & US & CT & MRI \\
\hline Spatial Resolution $(\boldsymbol{\mu m})$ & $150-200$ & 400.0 & $500-1000$ \\
\hline Penetration Depth $(\mathbf{n m})$ & 5 to 8 & NA & NA \\
\hline Lipid Pool & Fair & Good & Good \\
\hline Fibrous Cap & Fair & Poor & Fair \\
\hline Thrombus & Excellent & Excellent & Fair \\
\hline Activity & Fair & Poor & Good \\
\hline & & & \\
\hline Intraplaque Hemorrhate & No & No & Yes \\
\hline Neovasculature & No & No & Yes \\
\hline
\end{tabular}

US, ultrasound; CT, computed tomography; MRI, magnetic resonance imaging NA, not applicable $[63,70]$.

Table 1: Comparison of plaque imaging modalities. 


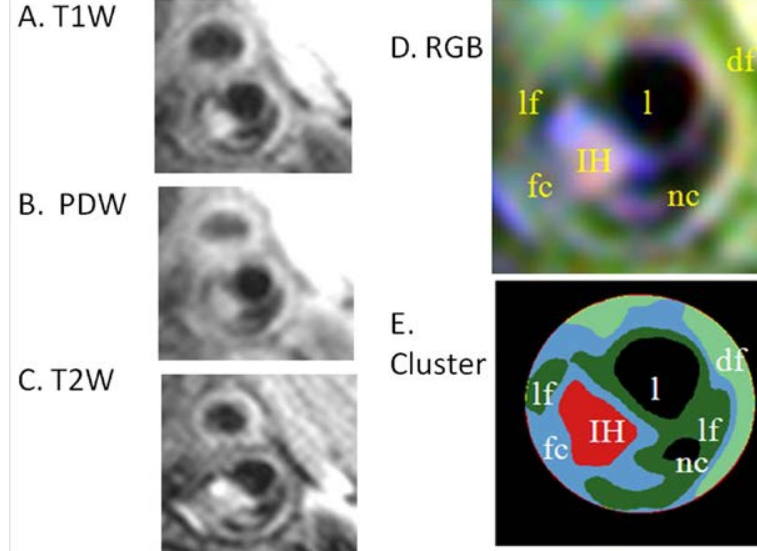

Figure 1: MRI of Intraplaque hemorrhage. A) T1W-hyperintense necrotic core with hemorrhage and isointense fibrous tissue B) PDW, C) T2W-iso/ hypointense necrotic core with hemorrhage and iso/hyperintense fibrous tissue, D) RGB image E) Cluster image.

\section{Contrast-Enhanced MRI (CE-MRI)}

One limitation of unenhanced MRI is the contrast-to-noise ratio (CNR) of the vessel wall that leads to lower resolution of vessel wall and plaque images. Contrast agents are used to improve CNR and provide better visualization of the vessel wall and plaque. A contrast agent, like gadolinium, accumulates in atherosclerotic plaques leading to increase in signal intensity. Zhang et al. demonstrated that contrastenhanced images correlate more closely to the histological specimen measurements of the wall compared to non-contrast images [37]. Barkhausen et al. also showed that CE-MRI could be used to visualize plaques not seen unenhanced images. It can also detect early-staged atherosclerosis in vessels where luminal narrowing is not yet seen. CEMRI, thus, leads to more accurate wall measurements and enhances imaging of atherosclerotic plaques [38].

These contrast agents are indirectly detected by their effect on the surrounding water protons. Application of the receptor-induced magnetization enhancement (RIME) phenomenon, where the contrast agent binds to the macromolecule of the tissue of interest significantly slowing down the molecular rotation of the contrast agent, such as gadolinium, permits for increased relaxivity and thus increased tissue contrast enhancement [39]. T1-shortening MR contrasts are predominately based on gadolinium. The molecular properties of gadolinium provide a spin relaxation time that is slow enough to allow for significant interaction with adjacent water protons. It has been shown that gadolinium-based contrast agents have a stronger effect on the shortening of $\mathrm{T} 1$ than $\mathrm{T} 2$ relaxation time [40]. This increase in signal intensity on T1-weighted images is termed positive contrast effect. While T2 relaxation time can also demonstrate considerable shortening, it has only observed in high concentrations.

Contrast-enhanced MRI allows for differentiation of plaque components which helps identify patients at risk for ischemic events. CE-MRI can enhance fibrous caps, allow their quantitative measurements, and distinguish intact from ruptured caps $[38,41,42]$. Quantitative measurement of fibrous caps is important because thin fibrous caps are more prone to rupture and this disruption of caps leads to embolism. The underlying collagen and lipid core of the ruptured plaque may also become exposed to blood components triggering the process towards thrombosis. Lipid-rich necrotic core (LRNC), can be assessed accurately by CE-MRI where it appears darker with contrast. The absence of vessels and matrix in this area of plaque results in minimal or no enhancement upon contrast administration. This difference in enhancement can be used to delineate LRNC and measure its size. Using CE-MRI T1-weighted imaging, the short-term regression or progression of LRNC in subclinical carotid atherosclerosis has been studied. Results showed that the presence of IPH appeared to be major factors in LRNC progression or regression and not statin therapy [43].

Gadolinium contrast can also be an indicator for inflammation and neovascularization. Papini et al. has been able to compare in vivo MRI images with cellular infiltrate on histology. Increase in contrast enhancement showed an increase in inflammation in histological specimens [44]. Both fibrous tissue and neovasculature have been reported to have higher signal enhancement than other plaque components, whereas the lipid-rich necrotic core tends to have a slower uptake and lower enhancement. Contrast-enhanced standard MRA technique has been shown to be highly sensitive for identifying IPH. A highly T1-weighted sequence with no increase in scan time yields results similar to specialized IPH techniques in regards to IPH detection [34].

T2-shortening MR contrast agents are predominantly based on iron oxide particles induce stronger $\mathrm{T} 2$ shortening compared to T1 relaxation times which results in a negative contrast effect which is detected as signal voids. Ultra-small super-paramagnetic iron oxide nanoparticles (USPIO) act as contrast media that demonstrate inflammation via macrophage imaging. USPIO are nanoparticles with an iron oxide core stabilized by a polymer coating that were initially used for imaging of the reticuloendothelial system, tumors, and inflammatory diseases of the central nervous system. Their application has expanded to include the imaging of atherosclerotic plaque [45]. Endothelial dysfunction during the atherosclerotic process triggers inflammation and lipid accumulation in the vessel wall. USPIO, due to their small size and water-solubility, extravasate through this inflamed and permeable endothelium. They accumulate in atherosclerotic plaques and, like lipid particles, are also taken up by macrophages. When macrophages internalize the iron particles, these particles generate their own magnetic fields that offset signals on T2-weighted images. The result is a signal loss that correlates with the amount of macrophage uptake and inflammation in the tissue [46].

A limitation of this technique is that signal diminution may also be caused by other factors such as calcified tissue and respiratory motion [46]. Ruptured atherosclerotic arteries also showed an increase accumulation of USPIO in rabbits compared to non-disrupted arteries [47]. In addition to ruptured arteries, Kooi et al. demonstrated that USPIOs are also predominant in vulnerable plaques in humans [48].

Macrophages consume USPIO via a non-specific receptormediated endocytosis. A high amount of contrast agent was administered to animal models to be able to have enough particles internalized by macrophages. To decrease the needed dose, studies have been done to increase the affinity of these cells to USPIO. Since macrophages in atherosclerotic plaque express mannose receptors, this has been studied as a way of increasing uptake and decreasing the dose. A study comparing four types of superparamagnetic iron oxides showed that mannan-coated SPIO and USPIO were more superior to unbound SPIO and USPIO in atherosclerosis imaging [49]. Tsuchiya et al. demonstrated that mannan-dextran coated USPIO is also more easily taken up by macrophages than dextran-USPIO [50].

MR molecular imaging with target specific molecular probes 
demonstrates the potential for non-invasive in vivo visualization of biological processes on the molecular and cellular level. There are several different classes of target-specific MR contrast agents available for molecular imaging. Molecular MR contrast agents, which enhance specific molecules (eg, elastin, fibrin, VCAM-1) or cells (eg, macrophages), allow visualization of pathologic processes on a molecular level with high spatial resolution; molecular MR imaging of atherosclerosis has the potential to improve early detection, guidance of treatment, and monitoring of treatment response. A fibrin-specific peptide conjugated to four gadolinium tetraazacyclododecane tetraacetic acid (FTCA, EPIX pharmaceuticals, Lexington, MA) successfully demonstrated intraplaque and endothelial fibrin imaging in vivo with molecular MRI after FTCA administration demonstrated a significant increase $(\mathrm{P}<0.05)$ in contrast agent uptake [51].

\section{Dynamic Contrast-Enhanced MRI}

Dynamic-contrast-enhanced (DCE) MRI is traditionally used in studying tumor microvasculature and angiogenesis but it was also served as a useful tool for quantification of the extent of plaque neovascularization [44]. DCE MRI can directly measure the effect of increased blood flow in the adventitia of newly formed vessels. In atherosclerotic plaque imaging, DCE-MRI is used to quantify adventitial vasa vasorum neovascularization and its permeability. This is done through determining kinetic parameters of contrast uptake such as measuring the transfer constant (vessel surface area and permeability) and blood flow [19]. The change in signal intensity is measured over time using T1W dynamic MR with high temporal resolution.

In DCE-MRI bright blood imaging utilizes the hyperintense signal emitted by flowing blood making it an ideal technique for assessing plaque microvessel density. In black blood imaging, blood flow and lumen signals are suppressed allowing for a more accurate vessel wall measurement and differentiation. Chen et al. was also able to use DCEMRI to study neovasculature and inflammation changes of early plaque lesions over time [52].

Calcagno et al. developed a new method to increase the sensitivity of DCE MRI in measuring neovascularization in atherosclerotic plaques. Parameters important in evaluating neovessels, vessel wall uptake and arterial input function, were evaluated using a simultaneous high and low spatial temporal resolution (SHILO) imaging technique. SHILO was validated against its standard counterpart, 2D spoiled gradient recalled echo (SPGR) acquisition, and results in both techniques were found to be similar. Having more consistent results compared to other widely used strategies, SHILO proved to be a leading technique in measuring DCE-MRI kinetic parameters to evaluate plaque vascularity and vessel wall permeability [53].

\section{Positron Emission Tomography-MRI}

At the forefront of non-invasive in vivo imaging of atherosclerosis is the use of positron emission tomography (PET) imaging in combination with MRI. PET assesses the metabolic activity of tissues and has been indispensable in the field of oncology. Its use has expanded to include investigation of atherosclerotic plaques. PET commonly uses 18-Fluorodeoxyglucose (FDG) as a tracer. FDG is a glucose analogue with a radioactive isotope, fluorine-18, at carbon 2. When inflammation increases the metabolism of glucose in tissues, FDG is taken up in cells the same way as glucose. Unlike its counterpart, FDG cannot be degraded and transported out of the cell. PET detects FDG accumulation in cells and measures it as SUV, standardized uptake value. SUV is increased in atherosclerotic plaques with unstable morphology and in sites of inflammation. These two are established factors that contribute to plaque rupture and subsequent acute thrombosis. Thus, PET imaging can quantify inflammation, tissue metabolism of glucose, and help in risk stratification of patients.

PET has a low spatial resolution that makes it susceptible to partial volume errors. To address this, it is usually co-registered with CT or MRI. PET will provide the information on metabolic activity and inflammation, while CT or MRI will allow for better visualization of anatomic structures and localization. Although promising, this system has several disadvantages. When patients are scanned in separate machines, lining up the anatomy in fusion programs can be burdensome. Putting the patient in the same position when scanning can also be challenging. In addition, PET makes use of isotopes that exposes patients to radiation. If coupled with CT, this will subject patients to additional exposure to radiation putting them at risk for cancer.

A combined PET-MRI machine for the imaging of atherosclerosis may prove to be a better alternative. MRI does not expose patients to radiation and has a more superior soft tissue contrast than CT. A study by Ripa et al. compared the performance of PET-CT vs PET-MRI in carotid arteries. Both were done to six HIV patients in less than an hour. SUV results between the two systems were found to be similar. PET-MR, however, was found to be better in differentiating the vessel wall. In addition, it is interesting to note that the study was done on patients without significant atherosclerotic plaques. Although certainly a limitation, this shows that the use of PET-MRI could be feasible in this type of patients who are in the early stages of atherosclerosis [54].

Taking a step further, Calcagno et al. studied the potential of PET and diffused contrast enhanced imaging (DCE-MRI) in assessing atherosclerotic plaque inflammation by detecting the presence of neovessels. Inflammation of atherosclerotic plaques has been linked to their subsequent neovascularization. Black blood sequence for DCE-MRI was utilized in the study to increase delineation of plaque characteristics. The results of the two imaging modalities were compared to a marker of inflammation, neovessel count, obtained from immunohistochemistry. Both PET and DCE-MRI were found to positively correlate with the neovessel count. Thus, these two imaging modalities could be useful for future studies in quantifying inflammation and detecting unstable atherosclerotic plaques [55].

\section{Clinical Applications of MR imaging based on Vascular Territories}

The risk of complications of atherosclerosis affects certain circulatory regions and yields distinct clinical manifestations depending on the particular circulatory bed affected. Atherosclerosis of the blood supply to the central nervous system (CNS), chiefly the carotid arteries, frequently incite ischemic stroke or transient cerebral ischemia. In the coronary arteries, myocardial infarction is commonly attributed to atherosclerosis. Mesenteric ischemia can be caused by atherosclerosis of the splanchnic circulation. Atherosclerosis in the peripheral arteries can cause claudications and jeopardize limb viability. This section will describe the current developments in MR imaging of atherosclerotic plaques and its clinical application to the most susceptible circulatory regions for thrombo-embolic events; carotids, aorta, coronary arteries, and peripheral arteries.

\section{Carotid Arteries}

Some of the most significant developments in MRI of atherosclerosis 
in recent years pertain to the baseline MRI characteristics of carotid atherosclerosis with clinically relevant outcomes. Features of carotid arteries such as superficial location and relative immobility make these vessels better suited for atherosclerotic plaque MR imaging than do other vessels such as the aorta or coronary arteries. Figure 2 show MR imaging techniques to assess plaque burden in carotid arteries. The measurements of carotid IMT-a strong predictor of future vascular events- by unenhanced T1-, T2- and PD carotid MR has been found comparable to that of carotid B-mode ultrasound [56].

Using MRI, studies show that type VI lesions with evidence of vulnerable plaque features, like IPH or cap rupture, have commonly been identified in carotid arteries with minimal to moderate stenosis [57]. An association between carotid IPH and cerebral ischemia was originally established by comparing in vivo preoperative imaging of atherosclerotic lesions with corresponding histological findings of carotid endarterectomy (CEA) specimens [58]. A strong association is reported between fibrous cap thinning/rupture, as determined by MRI TOF vessel wall imaging, and recent history of cerebrovascular events. $\mathrm{T} 2 \mathrm{~W}$ imaging of human carotid atherosclerotic plaque specimens has accurately detected advanced lesions type $\mathrm{Vb}$ fibrocalcific plaques [59]. Multi-contrast high resolution MRI can reliably identify intermediate to advanced atherosclerotic lesions and distinguish advanced lesions from early and intermediate in accordance with AHA classification [41].

There is abundant evidence that supports that MR imaging can display plaque vulnerability features i.e. IPH and fibrous cap disruption, in vivo in both asymptomatic and symptomatic cohorts of carotid stenosis. In a prospective study on patients initially asymptomatic with $50 \%$ to $79 \%$ carotid stenosis, arteries with thinned or ruptured fibrous caps, intraplaque hemorrhage, larger maximum percentage lipid-rich necrotic cores, and larger maximum wall thickness by MRI were associated with the occurrence of subsequent cerebrovascular events [60]. Multivariate cox regression analysis demonstrated a significant association between baseline MRI identification of the following plaque characteristics and subsequent symptoms during follow-up: presence of a thin or ruptured fibrous cap (hazard ratio, $17.0 ; \mathrm{P}<$ or $=0.001$ ), intraplaque hemorrhage (hazard ratio, 5.2; $\mathrm{P}=0.005$ ), larger mean intraplaque hemorrhage area (hazard ratio for $10 \mathrm{~mm} 2$ increase, 2.6; $\mathrm{P}=0.006$ ), larger maximum percentage lipidrich necrotic core (hazard ratio for $10 \%$ increase, $1.6 ; \mathrm{P}=0.004$ ), and larger maximum wall thickness (hazard ratio for a $1-\mathrm{mm}$ increase, 1.6; $\mathrm{P}=0.008$ ) [61]. Singh et al. conducted a cohort on asymptomatic moderate carotid stenosis showed that IPH - detected by rapid threedimensional T1-weighted fat suppressed gradient-echo sequence - was associated with an increase risk for ipsilateral cerebrovascular events. Of the 91 initially asymptomatic males with $50-70 \%$ stenosis, all six of the cerebrovascular events occurred in arteries with a baseline IPH (hazard ratio, 3.59; 95\% confidence interval: 2.48, 4.71; $\mathrm{P}<.001$ ) and MR-depicted IPH negatively predicted outcomes (negative predictive valued $=100 \%$ ) [60]. Among the 64 symptomatic patients with $30-60 \%$ stenosis followed for a mean period of 28 months, thirty nine (61\%) showed baseline IPH on MRI. Of those with baseline MRI-depicted IPH, 13 developed ipsilateral ischemic events, with only one reported TIA among those with no baseline IPH (hazard ratio, 9.8; $\mathrm{p}=0.03$ ) [62]. The presence of hyperintense signals on TOF, representing IPH, in a study of 112 patients for carotid artery stenting (CAS) showed a significantly higher likelihood of periprocedural symptoms $(18.4 \%$ vs $1.4 \%, p=0.0003$ ) [63]. Ota et al. used 3T MRI to show intraplaque hemorrhage and larger percentage of lipid-rich necrotic core are independently associated with thin or ruptured fibrous caps in patients with more than $50 \%$ carotid stenosis. This multivariate ordinal regression analysis demonstrated larger percentage of LRNC (odds ratio for $10 \%$ increase, $1.49 ; \mathrm{P}=0.02$ ) and presence of hemorrhage (odds ratio, 5.91; $\mathrm{P}<0.001$ ) were independently associated with a worse (intact thin or ruptured) stage of fibrous cap status. For artery-based multivariate analysis, a larger maximum percentage of LRNC and presence of hemorrhage independently associated with worse fibrous cap status $(\mathrm{P}<0.001$, for both) [64]. Hence, different MRI weightings can be used to determine the location, morphology, and composition of advanced plaque lesions.

High intensity signals observed in carotids plaque using inversion recovery-based 3D T1W imaging are associated with recent ischemic cerebrovascular events [65-67]. Carotid MR imaging has documented IPH using methemoglobin as an endogenous contrast agent resulting in a shortening of the $\mathrm{T} 1$ relaxation time and a hyperintense signal on T1W images. This technique, also termed as magnetizationprepared rapid acquisition with gradient echo (MPRAGE) or magnetic resonance direct thrombus imaging (MRDTI), can examine the components of complex AHA type VI plaques such as luminal surface defects, hemorrhage, thrombus, or calcified nodules $[10,68,69]$. MRDTI has been used to visualize intraplaque hemorrhage in patients with cerebral ischemia. The prevalence of AHA type VI carotid lesions using MRDTI in patients with varying degrees of carotid stenosis has been reported. The prevalence of high signal was significantly greater in the asymptomatic patients' ipsilateral vessels compared with the contralateral side $(60 \%$ versus $36 \%$, chi $2 \mathrm{P}<0.001)$, particularly for vessels of only moderate stenosis. The sensitivity and specificity of
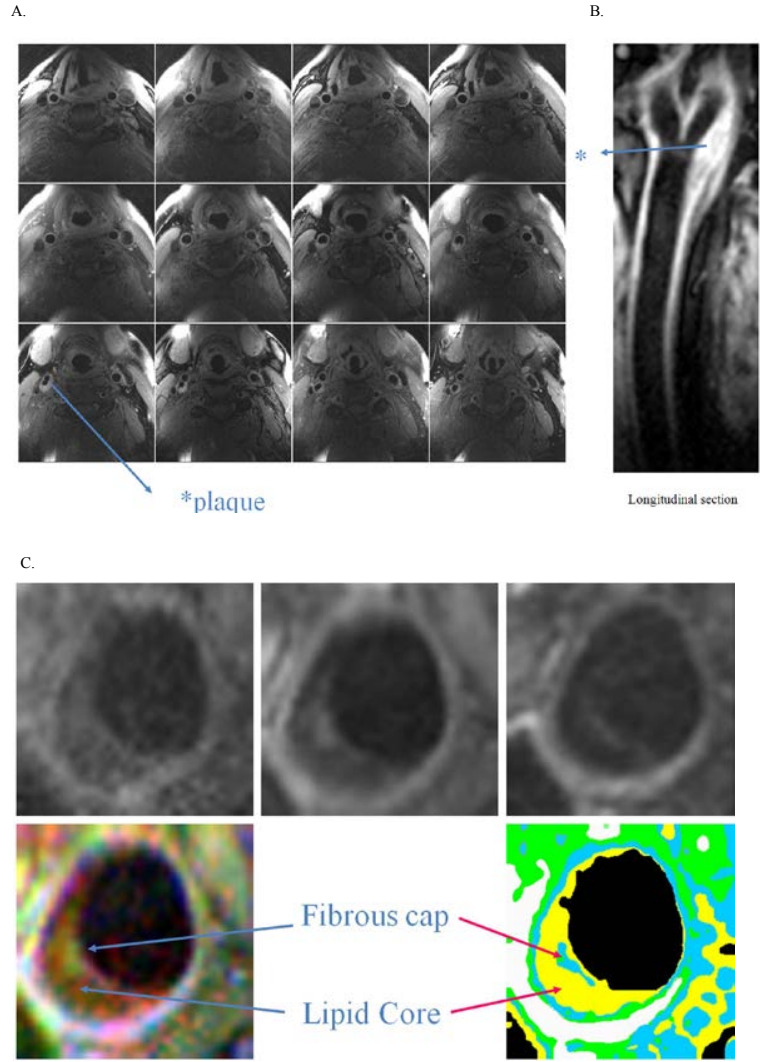

Figure 2: A) PDW images of carotid in 59 year old patients with atherosclerosis B) Longitudinal section of carotid plaques. * indicates plaque. C) Carotid plaque with vulnerable features of fibrous cap and lipid core. 
MRDTI for detecting complicated plaques was $84 \%$, with a positive predictive value (PPV) of $93 \%$, and a negative predictive value (NPV) of $70 \%$. There is also a significant negative correlation between the minimum luminal area and complicated AHA type VI. High degree stenosis (80-90\% luminal narrowing) had lesions with a LRNC was detected in $92 \%$ of all patients. The LRNC increased considerably with the higher degree of stenosis [69].

Carotid MRI studies demonstrate high level of reproducibility in assessment of plaque burden. Reproducibility of measurements on multiple scanner platforms has been established [70]. Plaque component identification has been widely validated and plaque composition has been accurately identified [38,41,71]. Fibrous cap, LRNC, hemorrhage, and thrombus have been identified on multicontrast MRI [43,61,69,72-74]. The accuracy and reproducibility of LRNC measurements via MRI show high sensitivity (85\%) and specificity (92\%), which has been utilize in the monitoring the plaque progression and regression [75-79].

Prospective MRI studies have also examined the role of components the atherosclerotic lesions in plaque progression. Takaya et al. showed that IPH, detected by HR MRI, is associated with greater plaque progression in both necrotic core and plaque volume [80]. IPH is shown to be an indication of accelerated plaque growth and luminal obstruction [81]. Sluimer et al., proposed that the destruction of the integrity of microvessel endothelium likely leads to intraplaque haemorrhage and plaques at increased risk for rupture [82]. Neoangiogenesis in atherosclerotic plaques has been implicated in the etiology of IPH, which when combined with LRNC, thin fibrous cap, and inflammation, can possibly serve as a good marker of vulnerable plaques. In recent years, plaque neoangiogenesis has become a target for asymptomatic vulnerable lesions and MRI is a noninvasive tool to further examine the underlying mechanisms. In terms of plaque regression in monitoring response to anti-atherosclerotic therapies, Corti et al. found a $15 \%$ reduction in the carotid wall area after 12 months of simvastatin treatment with no change is normal wall measurements [77]. Zhao et al. conducted a prospective assessment of MR carotid plaque composition during lipid lowering therapy and noted that after 3 years of lipid therapy, the 33 subjects with measurable LRNC at baseline had a significant reduction in plaque lipid content. Intensive lipid therapy significantly depletes carotid plaque lipid. Statistically significant plaque lipid depletion is observed after 1 year of treatment and continues in the second year, and precedes plaque regression [83].

\section{Aorta and Coronary Vessels}

There are numerous studies that assess and atherosclerotic burden in the thoracic and abdominal aorta. Thoracic aortic MR vessel wall imaging was found to be in good agreement with trans-esophageal echocardiography (TEE) in respect to plaque composition and thickness [29]. Figure 3 shows MR T1 and T2 images of atherosclerotic descending aorta. Although plaque extent in the aorta -both thoracic and abdominal- correlated with the severity of CAD, only thoracic plaques were independently associated with CAD [84].

In previous years, several reproducibility of studies of aortic wall measurements using multi-contrast MRI showed good inter-reader, intra-reader, and interscan reproducibility [85]. Since then, Calcagno et al showed good interscan and excellent intra- and interobserver reproducibility in an animal model of atherosclerosis using blackblood DCE MRI [86]. The application of DCE MRI monitoring high risk patients and in longitudinal clinical drug trials is currently being evaluated. Similar to carotid MR imaging, aortic vessel wall MR imaging studies directly visualize and quantify regression and progression of aortic atherosclerosis in response to different anti-atherosclerotic therapies.

A major challenge in MRI imaging of thoracic aorta is achieving sufficient sensitivity for submillimetre imaging and elimination of artifacts due to respiratory motion and pulsatile changes produced by blood flow. Such challenges have been addressed by technical
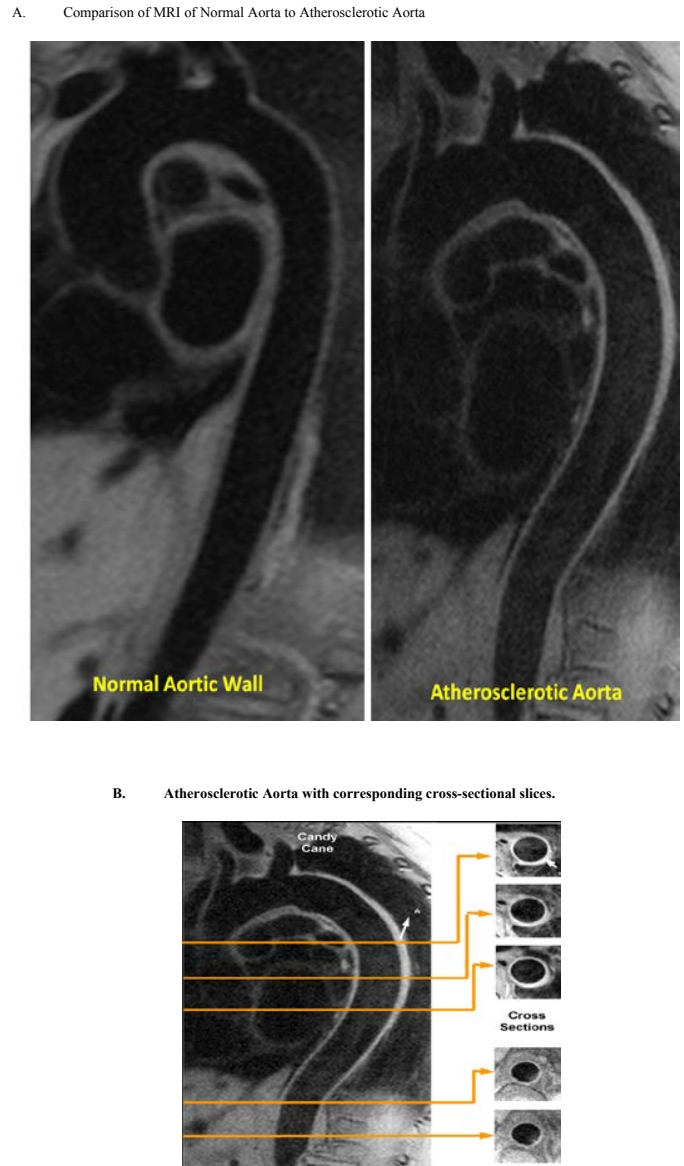

C. CMR of Aortic Plaque in T1 and T2 MRI

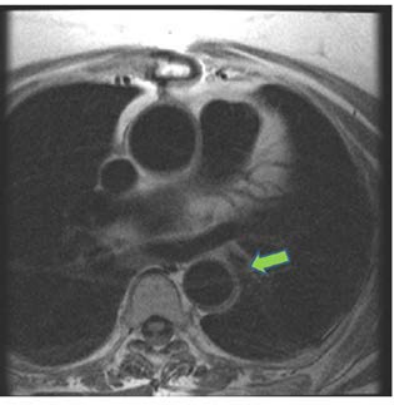

T1

Green Arrow indicates plaque

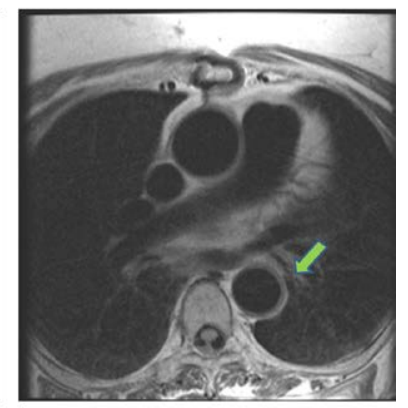

$\mathrm{T} 2$
Figure 3: MR imaging of Atherosclerosis of the Aortic Arch. A) Atherosclerotic Aorta depicting plaque in the arch is ulcerated resulting irregularity of the lumen. B) Aorta Candy cane view with corresponding cross sectional slices. C) CMR of Aortic Plaque in T1 and T2. 
improvements in magnetic field strength, dedicated sequences (such as double-inversion preparation pulses to suppress the signal of flowing blood), and navigation techniques to correct for respiratory motion [31].

As mentioned previously, MR imaging of coronary vessels is technically more challenging than other vascular beds. Coronary vessel wall imaging requires high resolution and high SNR due to the size of the vessel wall. Coronary imaging is further complicated by the need for cardiac and navigator gating. Respiratory compensation can be breath hold or free breathing with navigator echo monitoring the diaphragmatic motion. Recent improvements on navigator technology include ultrafast motion tracking, motion-adapted gating strategies, and the use of multiple navigators. The intrinsic motion of the coronary arteries during the cardiac cycle requires electrocardiographic synchronization of data acquisition [87].

Several studies support the coronary vessel MR imaging as a useful tool in detection of early atherosclerotic lesions in the coronary arteries. In a large asymptomatic multiethnic population, vessel wall MRI detected significant number of individuals with positive coronary remodeling with no history of CAD $[88,89]$. T2-weight black-blood coronary vessel wall MR imaging has been successful in detection and quantification of increased coronary vessel wall thickness and positive remodeling in patient with $\mathrm{CAD}$ confirmed by X-ray angiography. Coronary arterial plaques were first demonstrated in a porcine model using 2D T2W and PDW imaging sequences and validated with histopathology correlation [90].

Fayad et al. first demonstrated the feasibility of in vivo coronary plaque imaging using a spin echo black blood technique in humans [29]. Improvements in this technique allowed for free breathing through combining real-time navigator for respiratory gating and realtime slice-position correction [91]. High inter-study reproducibility has been established for serial evaluations of coronary vessel walls imaging and CAD [92]. Increased coronary wall thickness has been detected in patients with early CAD using MRI with isotrophic resolution [93].

Currently, coronary MR angiography (CMRA) is a promising noninvasive alternative for visualizing of coronary arteries. 3D steady-state free precession (SSFP) whole-heart CMRA is an unenhanced technique that permits visualization of all major coronary arteries with a single, axial 3D acquisition. Yoon et al demonstrated that CMRA is useful in predicting the risk of cardiac events in patients suspected to have CAD. Of the 207 patients with suspected coronary artery disease who underwent non-contrast-enhanced free-breathing whole-heart CMRA, there were 10 cardiac events- five of which were severe, observed in 84 patients with significant stenosis. In the 123 patients without significant stenosis, only 1 cardiac event with no severe event was observed. A significant difference in event-free survival between the 2 groups for severe events (annual event rate, 3.9\% and 0\%, respectively; log-rank test, $\mathrm{p}=0.003)$ was seen as well as for all cardiac events $(6.3 \%$ and $0.3 \%$; $\mathrm{p}<0.001)$. Cox regression analysis significant stenosis on CMRA was associated with a $>20$-fold hazard increase for all cardiac events (hazard ratio: 20.78 ; $95 \%$ confidence interval: 2.65 to $162.70 ; p=0.001$ ) [94].

Delayed-enhancement imaging of the coronary artery wall is another technique used which allows for direct assessment of contrast agents uptake in the vessel wall. In vivo, delayed enhancement showed nonspecific uptake in plaques in both patients with chronic angina and in those with acute coronary syndrome (ACS). Ibrahim et al. studied atherosclerotic plaque enhancement after acute myocardial infarction displayed coronary vessel wall contrast agent uptake is significantly increased early after myocardial infarction compared to those observed after 3 month follow up images [95]. This decrease in contrast uptake on follow up suggests uptake in patients ACS was more transient and more likely to be attributed to inflammation.

Due to the limited data on coronary vessel MR imaging, there is a need for more prospective data on these modalities to investigate the clinical role of these techniques for better diagnosis and characterization of CAD.

\section{Peripheral Arteries}

There have been some developments in MR imaging on disease of peripheral arteries. Angiography has been widely used for assessment of peripheral artery disease (PAD) caused by atherosclerosis [96]. Vessel areas and calcifications measured on high resolution TOA MRA were found to be in good agreement with IVUS measurement [97]. Measurement of plaque volume of the femoral artery has demonstrated interobserver, intraobserver, and test-retest reproducibility on black blood imaging [98]. Vessel wall measurement of femoral arteries is comparable in both 3D T2-weighted and 2D T1-weighted fast spin echo [99]. Due to poor spatial coverage, 2D imaging techniques for evaluation of PAD in vivo alternative MRI modalities have been studied to improve plaque detection and characterization lesions in the peripheral arteries. Hayashi et al showed that diffusion prepared dark blood 3D steady state free precession (3D-DP-SSFP) sequence indicated a higher signal to noise ratio (SNR) and higher contrast to noise ratio (CNR) compared to 2D-TSE technique. Figure 4 displays dark blood MRI protocol for femoral plaque imaging. This study showed excellent inter-observer reproducibility for 3D plaque burden [100]. In addition, Lui et al. showed improved arterial wall delineation in susceptibility weighted phase imaging of peripheral arterial walls [101].

Cross-sectional MRI analysis have shown to be a useful prognostic tool in monitoring plaque remodeling and restenosis. MR imaging has been applied in the development of restenosis of lower extremity bypass grafts by means of providing serial imaging of subjects to document progression of disease [102]. Efficacy of percutaneous transluminal angioplasty and its combination with endovascular brachytherapy, multicontrast double inversion recovery fast spin echo (DIR-FSE) was used to detect changes in vascular remodeling [103]

CE-MRA of the renal and aorto-iliac-femoral arteries has shown to detection significant steno-occlusive disease using different gadoliniumbased contrast agents. CE-MRA with gadobenate dimeglumine was more specific $(92.4 \%$ vs. $80.5 \%, \mathrm{p}<0.0001)$ and accurate $(83.6 \%$ vs. $77.1 \%, \mathrm{p}=0.022$ ) than CE-MRA with gadofosveset in the detection of significant renal artery stenosis. The average sensitivity was higher for gadofosveset $(74.4 \%$ vs. $67.3 \%, p=0.011)$ in peripheral vessels although gadobenate dimeglumine was more specific ( $93.0 \%$ vs. $88.2 \%$, $\mathrm{p}<0.0001)$ with no difference in accuracy $(86.6 \%$ vs. $86.3 \%, \mathrm{p}=0.66)$ PPVs were higher $(\mathrm{p}<0.0001)$ for gadobenate dimeglumine in both vascular territories. Pre- to post-test shifts in the probability of detecting significant disease were greater after gadobenate dimeglumine. Adverse events in the renal and peripheral studies were reported by $9.2 \%$ and $7.7 \%$ of patients after gadobenate dimeglumine compared with $30.3 \%$ and $22.1 \%$ of patients after gadofosveset [104].

In the largest comparative study to date, Hansmann et al. tested the diagnostic accuracy of time-resolved MRA of the calves compared to continuous-table movement MRA in symptomatic patients with lower extremity peripheral artery disease using digital subtraction angiography (DSA) as a reference standard. This study established 


\section{D TSE image}

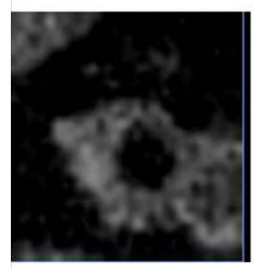

TWI
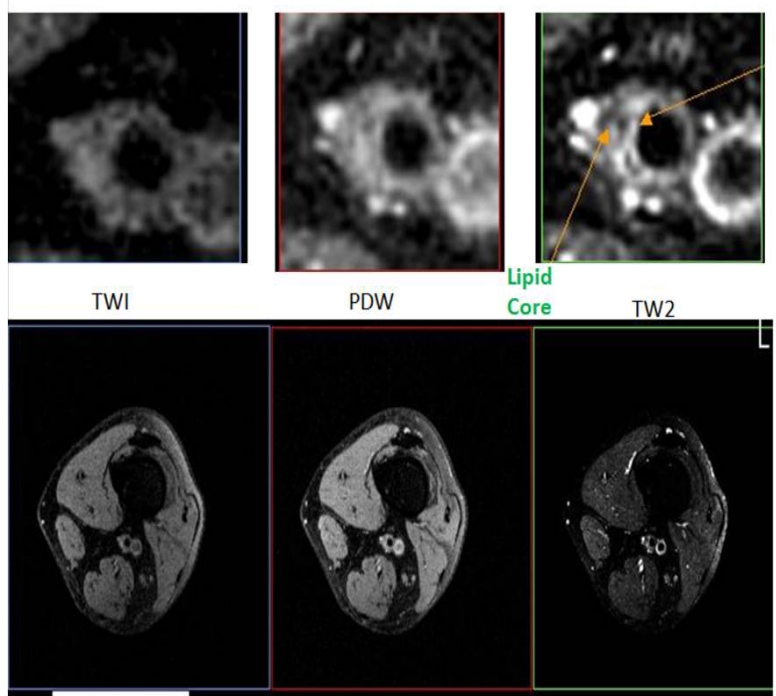

TW2

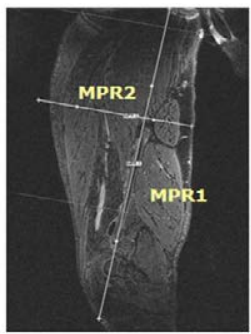

Multi planar reformatting

Diffusion SSFP

Femoral artery

1
B.

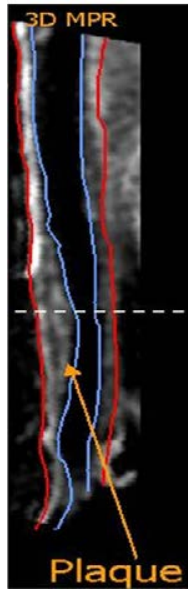

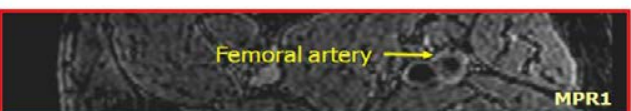

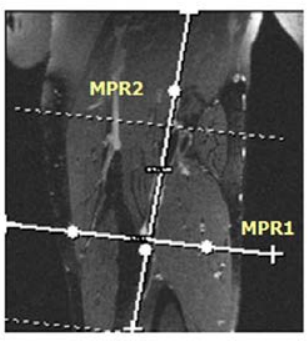

Multi planar reformatting

SPACE

Femoral artery
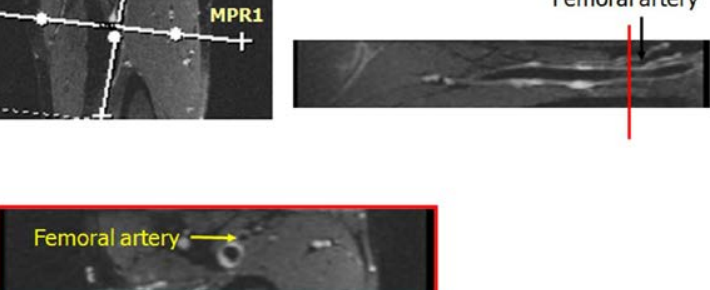

Figure 4: Dark blood MRI protocol for femoral plaque imaging. A) 2D black blood turbo spine echo (TSE) sequence using different contrast weightings to evaluate plaque composition in femoral artery. B) 3D Multi planar reformatting (MPR) of femoral artery with plaque indicated by orange arrow. C) MPR Diffusion SSFP. D) MRP SPACE.

that time-resolved MRA increases diagnostic accuracy of calf station. Median image quality of time-resolved MRA was rated excellent compared to continuous-table-movement MRA. Inter-reader agreement was excellent $(\kappa=0.80-0.84)$. The diagnostic accuracies (continuous-table-movement MRA/time-resolved MRA) combined for the readers were obtained for the tibioperoneal trunk $(84 \% / 93 \%)$, 
anterior tibial $(69 \% / 87 \%)$, posterior tibial $(85 \% / 91 \%)$, and peroneal $(67 \% / 81 \%)$ arteries. The advantages of time-resolved MRA include reduced venous contamination, improved arterial phase of contrast enhancement, and more precise delineation of vascular anatomy in comparison to continuous-table-movement MRA. This evidence supports the addition of time-resolved MRA to continuous-table MRA of the calf station as robust diagnostic approach for advanced PAD [105].

\section{Future Perspectives of MRI imaging of Atherosclerosis}

For asymptomatic patients with low-grade carotid artery stenosis, screening with a non-invasive in vivo imaging method like MRI can be a useful tool in detecting vulnerable atherosclerotic plaques. Plaque compositions that indicate instability have been identified. However, large clinical trials are needed to assess the direct clinical correlation of these plaque characteristics to cardiovascular and neurologic events. Selection criteria that are reliable and reproducible for identifying plaques at risk of causing an ischemic event are crucial in bringing this method of imaging to the clinical setting.

Temporal stability of atherosclerotic plaques is another aspect of atherosclerotic plaque analysis that requires further study. Clinical studies need longer follow-up times to determine the natural course of these plaques. There is a need for more definite clinical correlation and a better understand of the temporal evolution of vulnerable plaques which could improve risk stratification and management algorithmsincluding clinical trials on lipid lowering drugs, for those identified with early lesions of atherosclerosis. In the largest prospective study to date of patients with moderate to severe symptomatic carotid disease, Akram et al. determined the predictive power of intraplaque hemorrhage (IPH) for recurrent stroke with a follow-up of 9 years. 179 patients with previous cerebral ischemic events and more than 50\% carotid stenosis underwent brain and neck MRI imaging. 1.5 $\mathrm{T}$ scanners and standard quadrature neck array coils were used in the study. Intraplaque hemorrhage was found to strongly and independently predict secondary ipsilateral ischemic events in this subset of patients. Hosseini et al. also performed a meta-analysis of studies involving MRI imaging of patients with carotid stenosis. IPH was determined to be significantly associated with new and recurrent cerebral ischemic events for symptomatic patients. This shows that IPH is a possible biomarker for thromboembolic risk for patients who had previous ischemic events. For patients with asymptomatic carotid stenosis, however, it was found that available studies are limited with a small sample size and their allotted time for follow-up are inadequate to make any conclusion regarding the predictive value of IPH [106].

MRI imaging of atherosclerosis has also been studied in coronary arteries and other vessels. In the Multi-Ethnic Study of Atherosclerosis (MESA), compensatory enlargement of the arterial wall seen in early atherosclerosis was detected in patients without any history of coronary artery disease [89]. Coronary artery MRI imaging can present a major challenge because of the tortuosity of coronary arteries, cardiac and respiratory movement, and low spatial resolution. MRI imaging protocols that combine speed and resolution are being developed to overcome these inherent limitations of coronary imaging.

Since adequate non-invasive imaging of vulnerable atherosclerotic plaque in coronary arteries is still a work in progress, Wang et al. determined in a study that the carotid artery could be a window to the coronary arteries. Based on the premise that atherosclerosis is a systemic inflammatory disease of vessels in general, vulnerable carotid artery plaques may be used as a surrogate marker to identify patients at risk for acute coronary syndromes (ACS). In this study, ruptured carotid plaques are more common in patients with ACS compared to those with stable angina. Thus, ruptured carotid plaques may be used as an indication that patients are at risk for ACS [107]. More recently, carotid plaque vulnerability can also be useful in predicting future coronary events as well as cerebrovascular. Noguchi et al determined whether carotid high-intensity plaques (HIP) visualized by MPRAGE could be a predictor for coronary events. In 217 clinically stable CAD patients, the signal intensity of carotid plaques detected by MPRAGE and IMT measured by ultrasonography were examined and followed up for as long as 72 months. A carotid HIP was defined as a signal $>200 \%$ that of the adjacent muscle. They found a significant association between the presence of HIP and cardiac events compared to non-HIP (log-rank $\mathrm{P}<0.0001)$. Multivariate Cox regression identified the presence of HIP as the strongest independent predictor of cardiac events (hazard ratio: 3.15; $95 \%$ confidence interval: 1.93 to $5.58, \mathrm{p}<0.0001$ ) compared with IMT (hazard ratio: $1.62,95 \%$ confidence interval: 0.97 to $2.44, \mathrm{p}=0.055$ ) and other coronary risk factors [108]. Coronary imaging is a field of potential great impact for MRI imaging of atherosclerosis.

Currently, MRI imaging of atherosclerosis is limited to the field of research. For it to be applicable widely in a clinical setting, a standardized MRI protocol that maximizes visualization of the plaque and its components also needs to be established. Cost-effectiveness of the imaging technique is a concern. Training staff to employ the new protocol and analyze the images, obtaining dedicated coils, and the cost of MRI itself can be prohibitive for this imaging method to be a screening tool. Nevertheless, it will still be a more acceptable step compared to the invasive angiography procedures for asymptomatic patients.

\section{Conclusion}

Early assessment of atherosclerotic lesions is an important diagnostic goal in terms of decreasing the CAD burden. This review of MRI techniques of atherosclerosis provides an overview of the current MRI techniques and their clinical relevance. Magnetic resonance imaging is a well-established and reproducible technique that provides comprehensive information on the morphology, composition, and biochemical markers of atherosclerotic plaques and present high spatial resolution and good soft tissue contrast. Intraplaque hemorrhage and lipid rich necrotic core are considered the best indicators of vulnerable plaque lesions visualized by MRI. In addition, MRI imaging techniques offer other important information on the disease process like inflammation and neovascularization.

In the past decades, a great deal of technical improvements in MR imaging developments has been seen in carotid and aortic studies. Due to the technical difficulties of inherent motion coronary arteries assessment through MRI remains a challenge. However, data on coronary artery wall MR imaging has grown and provides useful clinical information plaque burden and positive arterial remodeling on therapeutic strategies and risk stratification. Research efforts are continually working to address these challenges. Developments in new cardiovascular imaging modalities provide the ability to track and quantify molecular biomarkers. Currently, molecular MR contrast agents and PET-MR are new fields of interests that show promising results on predicting clinical events and monitoring response to therapy. In conclusion, the rapid advancements of magnetic resonance imaging technology provide vast clinical opportunities for diagnosis, prevention, and treatment of atherosclerosis. 


\section{Acknowledgements}

The first and second authors would like to express their extreme gratitude to Dr. Venkatesh Mani for his patience.

\section{References}

1. Go AS, Mozaffarian D, Roger VL, Benjamin EJ, Berry JD, et al. (2013) Executive summary: heart disease and stroke statistics--2013 update: a report from the American Heart Association. Circulation 127: 143-152.

2. Iwakiri T, Yano Y, Sato Y, Hatakeyama K, Marutsuka K, et al. (2012) Usefulness of carotid intima-media thickness measurement as an indicator of generalized atherosclerosis: findings from autopsy analysis. Atherosclerosis 225: 359-362.

3. Falk E, Shah PK, Fuster V (1995) Coronary plaque disruption. Circulation 92 657-671.

4. Glagov S, Weisenberg E, Zarins CK, Stankunavicius R, Kolettis GJ (1987) Compensatory enlargement of human atherosclerotic coronary arteries. $\mathrm{N}$ Engl J Med 316: 1371-1375

5. Lindsay AC, Biasiolli L, Lee JM, Kylintireas I, Maclntosh BJ, et al. (2012) Plaque features associated with increased cerebral infarction after minor stroke and TIA: a prospective, case-control, 3-T carotid artery MR imaging study. JACC Cardiovascular imaging 5: 388-396.

6. Nasu K, Tsuchikane E, Katoh O, Vince DG, Margolis PM, et al. (2008) Impact of intramural thrombus in coronary arteries on the accuracy of tissue characterization by in vivo intravascular ultrasound radiofrequency data analysis. Am J Cardiol 101: 1079-1083.

7. Huang Y, Teng Z, Sadat U, He J, Graves MJ, Gillard JH (2013) In vivo MRIbased simulation of fatigue process: a possible trigger for human carotid atherosclerotic plaque rupture. Biomedical engineering online 12: 36 .

8. Virmani R, Burke AP, Kolodgie FD, Farb A (2003) Pathology of the thin-cap fibroatheroma: a type of vulnerable plaque. J Interv Cardiol 16: 267-272.

9. Stary HC, Chandler AB, Glagov S, Guyton JR, Insull W Jr, et al. (1994) A definition of initial, fatty streak, and intermediate lesions of atherosclerosis. A report from the Committee on Vascular Lesions of the Council on Arteriosclerosis, American Heart Association. Circulation 89: 2462-2478.

10. Stary HC, Chandler AB, Dinsmore RE, Fuster V, Glagov S, et al. (1995) A definition of advanced types of atherosclerotic lesions and a histological classification of atherosclerosis. A report from the Committee on Vascula Lesions of the Council on Arteriosclerosis, American Heart Association. Circulation 92: 1355-1374.

11. Sluimer JC, Daemen MJ (2009) Novel concepts in atherogenesis: angiogenesis and hypoxia in atherosclerosis. J Pathol 218: 7-29.

12. Moreno PR, Purushothaman KR, Sirol M, Levy AP, Fuster V (2006) Neovascularization in human atherosclerosis. Circulation 113: 2245-2252.

13. Kolodgie FD, Gold HK, Burke AP, Fowler DR, Kruth HS, et al. (2003) Intraplaque hemorrhage and progression of coronary atheroma. N Engl J Med 349: $2316-2325$

14. Rothwell PM, Eliasziw M, Gutnikov SA, Fox AJ, Taylor DW, et al. (2003) Analysis of pooled data from the randomised controlled trials of endarterectomy for symptomatic carotid stenosis. Lancet 361: 107-116.

15. McCarthy MJ, Loftus IM, Thompson MM, Jones L, London NJ, et al. (1999) Angiogenesis and the atherosclerotic carotid plaque: an association between symptomatology and plaque morphology. J Vasc Surg 30: 261-268.

16. Millon A, Mathevet JL, Boussel L, Faries PL, Fayad ZA, et al. (2013) Highresolution magnetic resonance imaging of carotid atherosclerosis identifies vulnerable carotid plaques. J Vasc Surg 57: 1046-1051

17. Xu X, Ju H, Cai J, Cai Y, Wang X, et al. (2010) High-resolution MR study of the relationship between superficial calcification and the stability of carotid atherosclerotic plaque. The international journal of cardiovascular imaging 26 Suppl 1: 143-150.

18. Gury-Paquet L, Millon A, Salami F, Cernicanu A, Scoazec JY, et al. (2012) Carotid plaque high-resolution MRI at $3 \mathrm{~T}$ : evaluation of a new imaging score for symptomatic plaque assessment. Magn Reson Imaging 30: 1424-1431.

19. Dong L, Kerwin WS, Chen H, Chu B, Underhill HR, et al. (2011) Carotid artery atherosclerosis: effect of intensive lipid therapy on the vasa vasorum-evaluation by using dynamic contrast-enhanced MR imaging. Radiology 260 224-231.
20. Corti R, Fuster V, Badimon JJ, Hutter R, Fayad ZA (2001) New understanding of atherosclerosis (clinically and experimentally) with evolving MRI technology in vivo. Ann N Y Acad Sci 947: 181-195.

21. Lorenz MW, Markus HS, Bots ML, Rosvall M, Sitzer M (2007) Prediction of clinical cardiovascular events with carotid intima-media thickness: a systematic review and meta-analysis. Circulation 115: 459-467.

22. Degnan AJ, Young VE, Gillard JH (2012) Advances in noninvasive imaging for evaluating clinical risk and guiding therapy in carotid atherosclerosis. Expert Rev Cardiovasc Ther 10: 37-53.

23. Fuji K, Hao H, Ohyanagi M, Masuyama T (2013) Intracoronary imaging for detecting vulnerable plaque. Circ J 77: 588-595.

24. Ferencik M, Nieman K, Achenbach S (2006) Noncalcified and calcified coronary plaque detection by contrast-enhanced multi-detector computed tomography: a study of interobserver agreement. J Am Coll Cardiol 47: 207-209.

25. Leber AW, Knez A, Becker A, Becker C, Rist C, et al. (2004) Accuracy of multidetector spiral computed tomography in identifying and differentiating the composition of coronary atherosclerotic plaques: a comparative study with intracoronary ultrasound. Journal of the American College of Cardiology 43 1241-1247.

26. Schroeder S, Kopp AF, Baumbach A, Kuettner A, Herdeg C, et al. (2001) Noninvasive detection of coronary lesions by multislice computed tomography: results of the New Age pilot trial. Catheter Cardiovasc Interv 53: 352-358.

27. Schroeder S, Kuettner A, Leitritz M, Janjen J, Kopp AF, et al. (2004) Reliability of differentiating human coronary plaque morphology using contrast-enhanced multislice spiral computed tomography: a comparison with histology. Journal of computer assisted tomography 28: 449-454

28. Achenbach S, Ropers U, Kuettner A, Anders K, Pflederer T, et al. (2008) Randomized comparison of 64-slice single- and dual-source computed tomography coronary angiography for the detection of coronary artery disease. JACC Cardiovasc Imaging 1: 177-186

29. Fayad ZA, Nahar T, Fallon JT, Goldman M, Aguinaldo JG, et al. (2000) In vivo magnetic resonance evaluation of atherosclerotic plaques in the human thoracic aorta: a comparison with transesophageal echocardiography. Circulation 101: 2503-2509.

30. Corti R, Wyttenbach R, Alerci M, Badimon JJ, Fuster V, et al. (2002) Images in cardiovascular medicine. Effect of percutaneous transluminal angioplasty on severely stenotic femoral lesions: in vivo demonstration by noninvasive magnetic resonance imaging. Circulation 106: 1570-1571.

31. Corti R, Fuster V (2011) Imaging of atherosclerosis: magnetic resonance imaging. Eur Heart J 32: 1709-1719b.

32. Gupta A, Baradaran H, Kamel H, Mangla A, Pandya A, et al. (2014) Intraplaque high-intensity signal on 3D time-of-flight MR angiography is strongly associated with symptomatic carotid artery stenosis. AJNR Am J Neuroradiol 35: 557-561.

33. Etesami M, Hoi Y, Steinman DA, Gujar SK, Nidecker AE, et al. (2013) Comparison of carotid plaque ulcer detection using contrast-enhanced and time-of-flight MRA techniques. AJNR Am J Neuroradiol 34: 177-184

34. Qiao Y, Etesami M, Malhotra S, Astor BC, Virmani R, et al. (2011) Identification of intraplaque hemorrhage on MR angiography images: a comparison of contrast-enhanced mask and time-of-flight techniques. AJNR Am J Neuroradiol 32: 454-459.

35. Anzalone N, Scomazzoni F, Castellano R, Strada L, Righi C, et al. (2005) Carotid artery stenosis: intraindividual correlations of 3D time-of-flight MR angiography, contrast-enhanced MR angiography, conventional DSA, and rotational angiography for detection and grading. Radiology 236: 204-213

36. Townsend TC, Saloner D, Pan XM, Rapp JH (2003) Contrast materialenhanced MRA overestimates severity of carotid stenosis, compared with 3D time-of-flight MRA. J Vasc Surg 38: 36-40.

37. Zhang S, Cai J, Luo Y, Han C, Polissar NL, et al. (2003) Measurement of carotid wall volume and maximum area with contrast-enhanced 3D MR imaging: initial observations. Radiology 228: 200-205.

38. Barkhausen J, Ebert W, Heyer C, Debatin JF, Weinmann HJ (2003) Detection of atherosclerotic plaque with Gadofluorine-enhanced magnetic resonance imaging. Circulation 108: 605-609.

39. Nivorozhkin AL, Kolodziej AF, Caravan P, Greenfield MT, Lauffer RB, et al. (2001) Enzyme-Activated $\mathrm{Gd}(3+)$ Magnetic Resonance Imaging Contrast 
Agents with a Prominent Receptor-Induced Magnetization Enhancement We thank Dr. Shrikumar Nair for helpful discussions. Angew Chem Int Ed Engl 40: 2903-2906.

40. Caravan P, Ellison JJ, McMurry TJ, Lauffer RB (1999) Gadolinium(III) Chelates as MRI Contrast Agents: Structure, Dynamics, and Applications. Chem Rev 99: 2293-2352.

41. Cai J, Hatsukami TS, Ferguson MS, Kerwin WS, Saam T, et al. (2005) In vivo quantitative measurement of intact fibrous cap and lipid-rich necrotic core size in atherosclerotic carotid plaque: comparison of high-resolution, contrastenhanced magnetic resonance imaging and histology. Circulation 112: 3437 3444.

42. Kwee RM, van Engelshoven JM, Mess WH, ter Berg JW, Schreuder FH, et al. (2009) Reproducibility of fibrous cap status assessment of carotid artery plaques by contrast-enhanced MRI. Stroke 40: 3017-3021.

43. Sun J, Balu N, Hippe DS, Xue Y, Dong L, et al. (2013) Subclinical carotid atherosclerosis: short-term natural history of lipid-rich necrotic core--a multicenter study with MR imaging. Radiology 268: 61-68.

44. Padhani AR, Hayes C, Landau S, Leach MO (2002) Reproducibility of quantitative dynamic MRI of normal human tissues. NMR Biomed 15: 143-153.

45. Ittrich H, Peldschus K, Raabe N, Kaul M, Adam G (2013) Superparamagnetic iron oxide nanoparticles in biomedicine: applications and developments in diagnostics and therapy. Rofo 185: 1149-1166.

46. Howarth SP, Tang TY, Trivedi R, Weerakkody R, U-King-Im J, et al. (2009) Utility of USPIO-enhanced MR imaging to identify inflammation and the fibrous cap: a comparison of symptomatic and asymptomatic individuals. Eur J Radio 70: 555-560.

47. Durand E, Raynaud JS, Bruneval P, Brigger I, Al Haj Zen A, et al. (2007) Magnetic resonance imaging of ruptured plaques in the rabbit with ultrasmall superparamagnetic particles of iron oxide. J Vasc Res 44: 119-128.

48. Kooi ME, Cappendijk VC, Cleutjens KB (2003) Accumulation of ultrasmall superparamagnetic particles of iron oxide in human atherosclerotic plaques can be detected by in vivo magnetic resonance imaging. Circulation 107: 24532458.

49. Tsuchiya K, Nitta N, Sonoda A, Otani H, Takahashi M, et al. (2013) Atherosclerotic imaging using 4 types of superparamagnetic iron oxides: new possibilities for mannan-coated particles. Eur J Radiol 82: 1919-1925.

50. Tsuchiya K, Nitta N, Sonoda A, Nitta-Seko A, Ohta S, et al. (2012) Evaluation of atherosclerotic lesions using dextran- and mannan-dextran-coated USPIO: MRI analysis and pathological findings. Int J Nanomedicine 7: 2271-2280.

51. Makowski MR, Forbes SC, Blume U, Warley A, Jansen CH, et al. (2012) In vivo assessment of intraplaque and endothelial fibrin in $\mathrm{ApoE}(-/-)$ mice by molecular MRI. Atherosclerosis 222: 43-49.

52. Chen H, Ricks J, Rosenfeld M, Kerwin WS (2013) Progression of experimental lesions of atherosclerosis: assessment by kinetic modeling of black-blood dynamic contrast-enhanced MRI. Magnetic resonance in medicine : official journal of the Society of Magnetic Resonance in Medicine / Society of Magnetic Resonance in Medicine 69: 1712-1720.

53. Calcagno C, Robson PM, Ramachandran S, Mani V, Kotys-Traughber M, et al. (2013) SHILO, a novel dual imaging approach for simultaneous HI-/LOW temporal (Low-/Hi-spatial) resolution imaging for vascular dynamic contrast enhanced cardiovascular magnetic resonance: numerical simulations and feasibility in the carotid arteries. J Cardiovasc Magn Reson 15: 42.

54. Ripa RS, Knudsen A, Hag AM, Lebech AM, Loft A, et al. (2013) Feasibility of simultaneous PET/MR of the carotid artery: first clinical experience and comparison to PET/CT. Am J Nucl Med Mol Imaging 3: 361-371.

55. Calcagno C, Cornily JC, Hyafil F, Rudd JH, Briley-Saebo KC, et al. (2008) Detection of neovessels in atherosclerotic plaques of rabbits using dynamic contrast enhanced MRI and 18F-FDG PET. Arterioscler Thromb Vasc Biol 28 1311-1317.

56. Underhill HR, Hatsukami TS, Cai J, Yu W, DeMarco JK, et al. (2010) A noninvasive imaging approach to assess plaque severity: the carotid atherosclerosis score. AJNR Am J Neuroradiol 31: 1068-1075.

57. Saam T, Underhill HR, Chu B (2008) Prevalence of American Heart Association type VI carotid atherosclerotic lesions identified by magnetic resonance imaging for different levels of stenosis as measured by duplex ultrasound. Journal of the American College of Cardiology 51: 1014-1021.
58. Imparato AM, Riles TS, Gorstein F (1979) The carotid bifurcation plaque: pathologic findings associated with cerebral ischemia. Stroke; a journal of cerebral circulation 10: 238-245.

59. Serfaty JM, Chaabane L, Tabib A, Chevallier JM, Briguet A, et al. (2001) Atherosclerotic plaques: classification and characterization with T2-weighted high-spatial-resolution MR imaging-- an in vitro study. Radiology 219: 403-410.

60. Singh N, Moody AR, Gladstone DJ, Leung G, Ravikumar R, et al. (2009) Moderate carotid artery stenosis: MR imaging-depicted intraplaque hemorrhage predicts risk of cerebrovascular ischemic events in asymptomatic men. Radiology 252: 502-508.

61. Takaya N, Yuan C, Chu B, Saam T, Underhill H, et al. (2006) Association between carotid plaque characteristics and subsequent ischemic cerebrovascular events: a prospective assessment with MRI--initial results. Stroke 37: 818-823.

62. Altaf N, Daniels L, Morgan PS (2008) Detection of intraplaque hemorrhage by magnetic resonance imaging in symptomatic patients with mild to moderate carotid stenosis predicts recurrent neurological events. J Vasc Surg 47: $337-$ 342.

63. Yoshimura S, Yamada K, Kawasaki M, Asano T, Kanematsu M, et al. (2011) High-intensity signal on time-of-flight magnetic resonance angiography indicates carotid plaques at high risk for cerebral embolism during stenting. Stroke 42: 3132-3137.

64. Ota H, Yu W, Underhill HR, Oikawa M, Dong L, et al. (2009) Hemorrhage and large lipid-rich necrotic cores are independently associated with thin or ruptured fibrous caps: an in vivo 3T MRI study. Arterioscler Thromb Vasc Biol 29: 16961701

65. Moody AR, Allder S, Lennox G, Gladman J, Fentem P (1999) Direct magnetic resonance imaging of carotid artery thrombus in acute stroke. Lancet 353: 122123.

66. Murphy RE, Moody AR, Morgan PS, Martel AL, Delay GS, et al. (2003) Prevalence of complicated carotid atheroma as detected by magnetic resonance direct thrombus imaging in patients with suspected carotid artery stenosis and previous acute cerebral ischemia. Circulation 107: 3053-3058.

67. Yamada N, Higashi M, Otsubo R, Sakuma T, Oyama N, et al. (2007) Association between signal hyperintensity on T1-weighted MR imaging of carotid plaques and ipsilateral ischemic events. AJNR Am J Neuroradiol 28: 287-292.

68. Leung G, Moody AR (2010) MR imaging depicts oxidative stress induced by methemoglobin. Radiology 257: 470-476.

69. Moody AR, Murphy RE, Morgan PS, Martel AL, Delay GS, et al. (2003) Characterization of complicated carotid plaque with magnetic resonance direct thrombus imaging in patients with cerebral ischemia. Circulation 107: 30473052.

70. Vidal A, Bureau Y, Wade T, Spence JD, Rutt BK, et al. (2008) Scan-rescan and intra-observer variability of magnetic resonance imaging of carotid atherosclerosis at 1.5 T and 3.0 T. Phys Med Biol 53: 6821-6835

71. Clarke SE, Hammond RR, Mitchell JR, Rutt BK (2003) Quantitative assessmen of carotid plaque composition using multicontrast MRI and registered histology. Magnetic resonance in medicine : official journal of the Society of Magnetic Resonance in Medicine / Society of Magnetic Resonance in Medicine 50: 11991208.

72. Mitsumori LM, Hatsukami TS, Ferguson MS, Kerwin WS, Cai J, et al. (2003) In vivo accuracy of multisequence MR imaging for identifying unstable fibrous caps in advanced human carotid plaques. J Magn Reson Imaging 17: 410-420

73. Yuan C, Kerwin WS, Ferguson MS, Polissar N, Zhang S, et al. (2002) Contrast-enhanced high resolution MRI for atherosclerotic carotid artery tissue characterization. J Magn Reson Imaging 15: 62-67.

74. Yuan C, Zhang SX, Polissar NL (2002) Identification of fibrous cap rupture with magnetic resonance imaging is highly associated with recent transient ischemic attack or stroke. Circulation 105: 181-185.

75. Yuan C, Mitsumori LM, Ferguson MS, Polissar NL, Echelard D, et al. (2001) In vivo accuracy of multispectral magnetic resonance imaging for identifying lipidrich necrotic cores and intraplaque hemorrhage in advanced human carotid plaques. Circulation 104: 2051-2056.

76. Corti R, Fayad ZA, Fuster V, Worthley SG, Helft G, et al. (2001) Effects of lipid-lowering by simvastatin on human atherosclerotic lesions: a longitudinal study by high-resolution, noninvasive magnetic resonance imaging. Circulation 104: 249-252. 
77. Corti R, Fuster V, Fayad ZA, Worthley SG, Helft G, et al. (2005) Effects of aggressive versus conventional lipid-lowering therapy by simvastatin on human atherosclerotic lesions: a prospective, randomized, double-blind trial with highresolution magnetic resonance imaging. J Am Coll Cardiol 46: 106-112.

78. Corti R, Fuster V, Fayad ZA, Worthley SG, Helft G, et al. (2002) Lipid lowering by simvastatin induces regression of human atherosclerotic lesions: two years' follow-up by high-resolution noninvasive magnetic resonance imaging. Circulation 106: 2884-2887.

79. Yonemura A, Momiyama Y, Fayad ZA, Ayaori M, Ohmori R, et al. (2009) Effect of lipid-lowering therapy with atorvastatin on atherosclerotic aortic plaques: a 2-year follow-up by noninvasive MRI. Eur J Cardiovasc Prev Rehabil 16: 222

80. Takaya N, Yuan C, Chu B, Saam T, Polissar NL, et al. (2005) Presence of intraplaque hemorrhage stimulates progression of carotid atherosclerotic plaques: a high-resolution magnetic resonance imaging study. Circulation 111: 2768-2775.

81. Underhill HR, Yuan C, Yarnykh VL, Chu B, Oikawa M, et al. (2009) Arterial remodeling in [corrected] subclinical carotid artery disease. JACC Cardiovasc Imaging 2: 1381-1389.

82. Sluimer JC, Kolodgie FD, Bijnens AP (2009) Thin-walled microvessels in human coronary atherosclerotic plaques show incomplete endothelial junctions relevance of compromised structural integrity for intraplaque microvascular leakage. Journal of the American College of Cardiology 53: 1517-1527.

83. Zhao XQ, Dong L, Hatsukami T, Phan BA, Chu B, et al. (2011) MR imaging of carotid plaque composition during lipid-lowering therapy a prospective assessment of effect and time course. JACC Cardiovasc Imaging 4: 977-986.

84. Taniguchi H, Momiyama Y, Fayad ZA, Ohmori R, Ashida K, et al. (2004) In vivo magnetic resonance evaluation of associations between aortic atherosclerosis and both risk factors and coronary artery disease in patients referred for coronary angiography. Am Heart J 148: 137-143.

85. Balu N, Wang J, Dong L, Baluyot F, Chen H, et al. (2009) Current techniques for MR imaging of atherosclerosis. Top Magn Reson Imaging 20: 203-215.

86. Calcagno C, Vucic E, Mani V, Goldschlager G, Fayad ZA (2010) Reproducibility of black blood dynamic contrast-enhanced magnetic resonance imaging in aortic plaques of atherosclerotic rabbits. Journal of magnetic resonance imaging 32: 191-198.

87. Makowski MR, Henningsson M, Spuentrup E, Kim WY, Maintz D, et al. (2013) Characterization of coronary atherosclerosis by magnetic resonance imaging. Circulation 128: 1244-1255

88. Gerretsen SC, Kooi ME, Kessels AG, Schalla S, Katoh M, et al. (2010) Visualization of coronary wall atherosclerosis in asymptomatic subjects and patients with coronary artery disease using magnetic resonance imaging. PLoS One 5 .

89. Miao C, Chen S, Macedo R (2009) Positive remodeling of the coronary arteries detected by magnetic resonance imaging in an asymptomatic population MESA (Multi-Ethnic Study of Atherosclerosis). Journal of the American College of Cardiology 53: 1708-1715

90. Worthley SG, Helft G, Zaman AG, Fuster V, Badimon JJ (2000) Atherosclerosis and the vulnerable plaque--imaging: Part II. Aust N Z J Med 30: 704-710.

91. Botnar RM, Stuber M, Kissinger KV, Kim WY, Spuentrup E, et al. (2000) Noninvasive coronary vessel wall and plaque imaging with magnetic resonance imaging. Circulation 102: 2582-2587.

92. Hazirolan T, Gupta SN, Mohamed MA, Bluemke DA (2005) Reproducibility of black-blood coronary vessel wall MR imaging. J Cardiovasc Magn Reson 7 : 409-413.

93. Kim WY, Stuber M, Börnert P, Kissinger KV, Manning WJ, et al. (2002) Threedimensional black-blood cardiac magnetic resonance coronary vessel wall imaging detects positive arterial remodeling in patients with nonsignificant coronary artery disease. Circulation 106: 296-299.
94. Yoon YE, Kitagawa K, Kato S, Ishida M, Nakajima H, et al. (2012) Prognostic value of coronary magnetic resonance angiography for prediction of cardiac events in patients with suspected coronary artery disease. Journal of the American College of Cardiology 60: 2316-2322.

95. Ibrahim T, Makowski MR, Jankauskas A, Maintz D, Karch M, et al. (2009) Serial contrast-enhanced cardiac magnetic resonance imaging demonstrates regression of hyperenhancement within the coronary artery wall in patients after acute myocardial infarction. JACC Cardiovascular imaging 2: 580-588.

96. Attenberger UI, Haneder S, Morelli JN, Diehl SJ, Schoenberg SO, et al. (2010) Peripheral arterial occlusive disease: evaluation of a high spatial and temporal resolution 3-T MR protocol with a low total dose of gadolinium versus conventional angiography. Radiology 257: 879-887.

97. Meissner OA, Rieger J, Rieber J, Klauss V, Siebert U, et al. (2003) Highresolution MR imaging of human atherosclerotic femoral arteries in vivo: validation with intravascular ultrasound. J Vasc Interv Radiol 14: 227-231.

98. Isbell DC, Meyer CH, Rogers WJ, Epstein FH, DiMaria JM, et al. (2007) Reproducibility and reliability of atherosclerotic plaque volume measurements in peripheral arterial disease with cardiovascular magnetic resonance. J Cardiovasc Magn Reson 9: 71-76.

99. Zhang Z, Fan Z, Carroll TJ, Chung Y, Weale P, et al. (2009) Three-dimensional T2-weighted MRI of the human femoral arterial vessel wall at 3.0 Tesla. Invest Radiol 44: 619-626.

100. Hayashi K, Mani V, Nemade A, Silvera S, Fayad ZA (2010) Comparison of 3D-diffusion-prepared segmented steady-state free precession and 2D fast spin echo imaging of femoral artery atherosclerosis. Int J Cardiovasc Imaging 26: 309-321.

101.Liu Q, Fan Z, Yang Q, Li D (2012) Peripheral arterial wall imaging using contrast-enhanced, susceptibility-weighted phase imaging. J Comput Assis Tomogr 36: 77-82.

102. Heverhagen JT, Kalinowski M, Schwarz U, Klose KJ, Alfke H (2000) Quantitative human in vivo evaluation of high resolution MRI for vessel wal morphometry after percutaneous transluminal angioplasty. Magn Reson Imaging 18: 985-989.

103. Wyttenbach R, Corti R, Alerci M, Cozzi L, Di Valentino M, et al. (2007) Effects of percutaneous transluminal angioplasty and endovascular brachytherapy on vascular remodeling of human femoropopliteal artery: 2 years follow-up by noninvasive magnetic resonance imaging. Eur J Vasc Endovasc Surg 34: $416-423$.

104. lezzi R, Soulez G, Thurnher S, Schneider G, Kirchin MA, et al. (2011) Contrastenhanced MRA of the renal and aorto-iliac-femoral arteries: comparison of gadobenate dimeglumine and gadofosveset trisodium. Eur J Radiol 77: 358368.

105. Hansmann J, Michaely HJ, Morelli JN, Diehl SJ, Meyer M, et al. (2013) Impact of time-resolved MRA on diagnostic accuracy in patients with symptomatic peripheral artery disease of the calf station. AJR Am J Roentgenol 201: 13681375 .

106. Hosseini AA, Kandiyil N, Macsweeney ST, Altaf N, Auer DP (2013) Carotid plaque hemorrhage on magnetic resonance imaging strongly predicts recurrent ischemia and stroke. Ann Neurol 73: 774-784.

107. Wang Q, Zeng Y, Wang Y, Cai J, Cai Y, et al. (2011) Comparison of carotid arterial morphology and plaque composition between patients with acute coronary syndrome and stable coronary artery disease: a high-resolution magnetic resonance imaging study. The international journal of cardiovascular imaging 27: 715-726.

108. Noguchi T, Yamada N, Higashi M, Goto Y, Naito H (2011) High-intensity signals in carotid plaques on T1-weighted magnetic resonance imaging predict coronary events in patients with coronary artery disease. Journal of the American College of Cardiology 58: 416-422. 